\title{
EVALUATION OF TOPICAL APPLICATION OF PLATELETS RICH PLASMA AND HYALURONIC ACID ON THE HEALING OF EXPERIMENTALLY INDUCED TONGUE ULCER
}

\author{
Asmaa S. Elgamal * and Laila E. Amin **
}

\begin{abstract}
Objectives: The current study intended to evaluate the effect of topical application of platelets rich plasma and hyaluronic acid on the healing of experimentally induced tongue ulcer.

Materials and methods: 80 white albino rats were used in the study; platelets rich plasma was isolated from 8 rats, by cardiac puncture, then mixed with orabase to form an oral gel. The remaining 72 rats were subjected to tongue ulcer induction. The rats then were divided into: Control group: ulcers were left without any treatment. hyaluronic acid group: ulcers were treated with topical applications of hyaluronic acid gel. platelets rich plasma group: ulcers were treated with topical applications of platelets rich plasma gel. Specimens were taken after 3rd, 7th, and 10th day and processed for histological and immunohistochemical staining for CD31 and KI67.
\end{abstract}

Results: Both platelets rich plasma and hyaluronic acid treated groups showed smaller ulcer size and faster healing histologically and immunohistochemically. The statistical analysis of CD 31 and Ki-67 immunohistochemical reaction along examination period showed significant difference between control group and hyaluronic acid \& platelets rich plasma group. Also, there was a significant difference between hyaluronic acid and platelets rich plasma groups at $3^{\text {rd }}$ and $7^{\text {th }}$ day. But, there was non- significant difference between hyaluronic acid \& platelets rich plasma groups at $10^{\text {th }}$ day.

Conclusion: the topical application of platelets rich plasma and hyaluronic acid can accelerate wound healing. PRP showed significant improvement of ulcer healing than HA by stimulating angiogenesis and induction of cellular proliferation.

KEY WORDS: Oral ulcers; Platelet Rich Plasma; Hyaluronic Acid; CD31and Ki67.

* Lecturer at Oral Biology Department, Faculty of Dentistry, Beni Suef University, Egypt.

** Associate professor of Oral Biology Department, Faculty of Dentistry, Mansoura University, Associate professor of Oral Biology Department, Faculty of Dentistry, Horus University, Egypt. 


\section{INTRODUCTION}

Traumatic ulcers are mostly frequent in the oral cavity and are an outcome of accidental damages. Traumatic ulcers are commonly originating in many mucosal surfaces as the cheek mucosa, tip of the tongue, lips, gingiva and soft palates. The oral ulcers are known signs in a variety of disorders that diverge from auto-immune diseases to malignant neoplasia and infective diseases, dietary insufficiencies, traumatic and allergic responses ${ }^{(1)}$.

Wound healing is a regular biological process and a very complex procedures in the human body. It is consisted of accurately programmed phases involving inflammation, cell proliferation, wound contraction, angiogenesis, matrix remodeling and epithelialization. Although the variability of topical treatments with non-steroidal anti-inflammatory agents, antihistamines, local anesthetics, and antibiotics used to relief the symptom, these agents have not been exactly assessed ${ }^{(2)}$.

Hyaluronic acid (HA) is a linear polymer of glucuronic acid and $\mathrm{N}$-acetylglucosamine disaccharide. The central role of HA through healing processes are the triggering of the inflammatory reactions, increase the cellular proliferation, migration, and angiogenesis. HA is a hygroscopic macromolecule and its solutions are highly osmotic. In the oral mucosa, this character permits to regulate the hydration of tissue throughout the inflammation during ulcer formation ${ }^{(3)}$. To understand the role of HA size in homeostasis and cancer, it is essential to distinguish these various molecular weight forms of high molecular weight HA (HMWHA, >1,000 kDa), medium molecular weight HA (MMW-HA, 250-1,000 $\mathrm{kDa}$ ), low molecular weight HA (LMW-HA, 10$250 \mathrm{kDa}$ ), and oligomeric HA (oligo-HA, <10 kDa) (4). Studies reveal that most of HA's properties are size-dependent. High molecular weight HA (HMWHA) exerts anti-inflammatory, immunosuppressive and wound healing effects, while low molecular weight HA (LMW-HA) shows pro-inflammatory properties ${ }^{(5)}$.

The regenerative medicine is developing as an alternative line to the traditional methods used in tissue repair through emerging the perception of addition the growth factors straight to the wound (6). Platelets rich plasma (PRP) is an autologous concentration of human platelets over the standard in a minor amount of plasma. Its mechanism of actions depends on the construction and liberation of various growth factors. PRP gel is used extensively in encouraging the repair of chronic cutaneous refractory wounds. Furthermore, it accelerates bone formation in the surgery field besides the treatment of numerous hard tissue defects ${ }^{(7)}$.

Topical treatment with autologous PRP considered a very important option in several clinical situations. PRP promote the production of great quantities of growth factors, which encourage the construction of collagen and extracellular matrix via least amounts of plasma. Glycoprotein PDGF is the main growth factor to first appear in the wound, initiating the repair process of the connective tissue. Its greatest significant actions comprise mitogenesis, angiogenesis, and macrophage activation ${ }^{(8)}$.

Proper wound healing necessitates the development of a granulation tissue with numerous of new blood vessels to preserve the nourishment of the wound area and support the deposition of extracellular matrix ${ }^{(9)}$. CD31 or Platelet-endothelial cell adhesion molecule is expressively expressed on the surface of the adult and embryonic endothelial cells. CD31 is critical for angiogenesis owing to its important character in the adhesion among endothelial cells and is considered one of the highest valued markers that detect endothelial cells ${ }^{(10)}$.

Oral mucosa is consisted of stratified squamous epithelium (SSE); the stratification is the end result of cell proliferation and progressive differentiation. Proliferation is a character of stem cells of the basal 
layers of the SSE and the transit-amplifying cells. Ki67 antigen is the best related immunohistochemical marker to detect cell proliferation is. Its nuclear expression during a distinct phase of the cell cycle denotes a benefit in its usage as a biological marker of mitotic activity ${ }^{(11)}$. Ki-67 protein is identified in $\mathrm{G} 1, \mathrm{~S}, \mathrm{G} 2$ and $\mathrm{M}$ phases of cell cycle, but not in G0. It can be used as a proliferation index as it is existing in all dividing cells of normal and tumor tissues, but absent in resting cells, and it is expressed only by dividing cells ${ }^{(12)}$.

Many authors used different types of platelets concentrates in treatment of different types of ulcers and fount significant improvement in healing process by using it. It was found that Local injection of PRP enhance the quality of the healing process of induced oral ulcer in Albino Rats ${ }^{(13)}$.

Moreover, Clinically, topical application of autologous platelets rich plasma promoted healing process of dormant corneal ulcers even in eyes threatened by corneal perforation ${ }^{(14)}$, oral erosions and ulcers induced by pemphigus vulgaris ${ }^{(15)}$ and chronic skin ulcer in AIDS patients ${ }^{(16)}$.

Ahmed et al., 2019 found human platelet lysate (HPL) had a superior effect when compared to corticosteroids, Clobetasol Propionate, in treatment of Methotrexate-induced oral ulcers in rheumatoid arthritis patients ${ }^{(17)}$. Ramzy et al., also reported that a single injection of PRP could be used as a therapeutic to enhance wound healing in diabetic and non-diabetic oral mucosal wounds ${ }^{(18)}$.

So, the present study suggested that topical application of PRP gel may improve the healing of oral ulcer. Thus, our objectives were the assessment of the result of topical use of PRP gel on the healing of experimentally induced traumatic tongue ulcer compared with commercially used hyaluronic acid gel (Gengigel). The healing process was evaluated through, histological and immunohistochemical examinations.

\section{MATERIALS AND METHODS}

\section{Study design}

The animal handling and experimental protocols was permitted according to ethical committee for animal care and followed to the roles defined the controlling principle for the animals' laboratory procedure in faculty of Dentistry, Mansoura University, Egypt.

The current study used 80 adult male Albino rats weighting from 150-200 gm, the animals were housed in separate cages under the optimal experimental conditions according to the guidelines of the Animal Ethics Committee. They were fed on ground barely and supplied water add-libitum. After one week acclimatization period, the animals were divided into:

\section{A) 8 rats were used for PRP isolation}

\section{B) 72 rats $(n=8)$ were subjected to ulcer induction and divided into}

I) Control group: ulcers were healing normally.

II) HA group: ulcers were treated with 3 times/ day topical applications of commercially used $0.2 \%$ high molecular weight hyaluronic acid.

III) PRP group: ulcers were treated with 3 times/ day topical applications of PRP gel.

\section{Preparation of PRP gel}

Blood was drained directly by cardiac puncture after deeply anaesthetized rats and inserted into a sodium citrate tube $(3.2 \%)$ which is anti-coagulant that keeping the blood from clotting. For PRP separation, the samples then centrifuged at 800 RPM for $15 \mathrm{~min}$ for. The average platelet count was $647 \times 103 / \mu \mathrm{L}^{(19)}$.

\section{Orabase preparation}

The orabase was performed in a pharmacy laboratory with the subsequent components: $16.6 \%$ gelatin, $16.6 \%$ pectin, $16.6 \%$ sodium carboxymethyl 
cellulose, $100 \%$ plasti-base (emollients topical. Bristol-Myers Squibb. Canada). This mucoadhesive paste was prepared by gradual addition of sodium carboxymethyl, cellulose, pectin and gelatin to the plastic-base and constant stirring to obtain a homogeneous base. The final formulation of PRP gel was prepared by mixing Orabase with PRP by ratio $1: 1(\mathrm{w} / \mathrm{w})$. This formulation was preserved at $4^{\circ} \mathrm{C}$ for a maximum of 14 days ${ }^{(19)}$.

\section{HA preparation}

Study material used was a commercially used hyaluronic acid gel; Gengigel ${ }^{\circledR}$ (via Egadi,7 -20144 Milano IT). The active ingredient in it is a High Molecular Weight (HMW) hyaluronic acid in a form of sodium hyaluronate. The other ingredients are: Aqua, Xylitol, Celleluse Gum, Alcohol, Peg 40 Hydrogenated Castor Oil, Dichlorobenzyl Alcohol, Carbomer, Aroma, Citric Acid, Sodium Hydroxide And Acidic Blue.

\section{Ulcer induction}

Rats were anaesthetized with an intramuscular injection of ketamine hydrochloride $10 \%$ and xylazine hydrochloride 2\% (AMOUN, Egypt) at a dosage of $0.06 \mathrm{ml} / 100 \mathrm{~g}$ and $0.03 \mathrm{ml} / 100 \mathrm{~g}$, correspondingly. A uniform regular Circular ulcer, with $4 \mathrm{~mm}$ diameter and $2 \mathrm{~mm}$ depth, on the dorsal surface of the tongue was done by $4 \mathrm{~mm}$ biopsy punch subsequently upright application of a swab with a cotton tip saturated in formocresol for 60s. The induced ulcers were left to heal either normally or topically covered with PRP orabase or $0.2 \%$ HA gel $\left(\right.$ Gengigel $\left.^{\circledR}\right)$. The rats were kept under observation without any feeding or drinking for I hour after topical application of treatments. Finally, the rats were scarified by overdose of sodium thiopental at day $3,7,10$ respectively.

\section{Histological examination}

Tongues from each animal were excised and immediately fixed in $10 \%$ formaldehyde with $\mathrm{PH}$
7.2 prepared in phosphate buffer saline (PBS) for 24 hours. Tissue processing and staining was performed in histopathological lab at faculty of Veterinary Medicine, Beni Siuef University. The tissue processing was completed using an automatic tissue processor which took the tissue through a series of graded alcohol baths and then into xylene. The processed tissues were embedded in paraffin blocks. Serial sections (4 microns thick) were obtained from each paraffin block, mounted on coated slides and dried for H\&E stain and immunohistochemical examination.

\section{Immunohistochemical staining}

Immunohistochemical study was done to detect the Ki-67 nuclear antigen to evaluate the cellular proliferation and CD31 to detect the endothelial cells proliferation. Immunohistochemical comprised the following stages ${ }^{(20,21)}$ :

Sections were mounted on silan-coated glass slides, deparaffinized with xylol and then rehydrated. Endogenous peroxidase was blocked using $\mathrm{H}_{2} \mathrm{O}_{2}$ at $3 \%$. Antigen retrieval was done with citrate buffer $0.01 \mathrm{M}$ ( $\mathrm{PH} \mathrm{6.0)}$. Slides were incubated with the primary rabbit anti human Ki-67 monoclonal antibody (Spring Bioscience, Pleasanton, California, USA) and the anti human CD31 monoclonal antibody (Santa Cruz, Biotechnology, California, USA). The slides were incubated with secondary biotinylated antibody and then incubated with complex streptavidin-biotin peroxidase. Development with chromogen substrate diaminobenzidine $(\mathrm{DAB})$ was done and finally the slides were counterstained with Harris hematoxylin. After each step the sections were washed with phosphate buffered saline (PBS). Negative controls were obtained using non-immune serum instead of the primary antibody. Slides were dehydrated and set up to be analyzed by optical microscopy.

It was considered to be positive nuclear reaction for Ki-67 by expression of brownish staining 
restricted to the nucleus ${ }^{(22)}$. Large and small vessels that were CD31-positive reaction (blood vessels) had developed and were concentrated at the submucosa and lamina propria of the lingual mucosa ${ }^{(23)}$.

\section{Morphometric and Statistical analysis}

Analysis of samples was performed by two double-blinded oral pathologists, previously standardized and calibrated. All samples were observed under an optical microscope (Olympus ${ }^{\circledR}$ CX-31). Slides were digitized using Olympus® digital camera installed on Olympus ${ }^{\circledR}$ microscope with $1 / 2 \mathrm{X}$ photo adaptor, using $40 \mathrm{X}$ objective. The result images were analyzed on Intel ${ }^{\circledR}$ Core $I 3 \AA$ based computer using Video Test Morphology® software (Russia) with a specific built-in routine for immunohistostaining analysis and stain quantification.

Percentage of vascular stain of CD31: For the vascular quantification, Morphometric Analysis System (SAMM), a computer program developed specifically for this purpose, was used. A positive reaction for $\mathrm{CD} 31$ was considered by the presence of brown colored immunostaining reaction in the cytoplasm and cell membrane of endothelial cells and stromal connective tissue cells. Sixteen random fields were analyzed for each group (two sections from each sample). The system was previously calibrated to recognize the color spectrum on the structure of interest (micro vessels), according to the technique used for staining. This procedure enables the software to automatically identify and target the blood vessels (separating them from the other components of the preparation). After the segmentation, the software determined the percentage of stained surface in the field. The calculation of area density was performed, which was defined by the ratio between the area occupied by microvasculature and the total area of the analyzed field ${ }^{(24)}$.

The number of Ki67-positive cells was measured by using the same software. The Ki- 67 labeling index (LI) for cell proliferation was used five areas at high power ( $\times 400$ magnification). A minimum of 500 cells were calculated, and the percentage among the number of cells that had a brown stained nucleus and the whole number of cells was calculated (20).

The data were examined consuming the Statistical Package for the Social Sciences (SPSS, version 16) software. Statistics were conveyed in the form of mean and standard deviation; the different groups were compared using ANOVA (F-test). ANOVA was followed by a post hoc test. Significance was considered at a $p$ value $<0.05$

\section{RESULTS}

\section{Histological analysis}

\section{1- Hematoxylin and Eosin stain}

At $3^{\text {rd }}$ day post ulceration: control group revealed discontinuity of the epithelium, formation of granulation tissue with severe inflammatory cell infiltration. HA group showed the ulcer area filled with inflammatory cells within granulation tissue. PRP groups revealed slight proliferation of the covering epithelium and formation of the granulation tissue with dilated blood vessels, and dense inflammatory cells infiltration (Figure 1 A, B, C).

At $7^{\text {th }}$ day: control group showed slight proliferation of the covering epithelium and formation of the granulation tissue with dense inflammatory cell infiltration. HA group showed partial healing process appeared through the proliferation of the covering epithelium, the involving cellular connective tissue stoma appeared with large number of fibroblasts, collagen fibers, newly formed blood vessels and decreased inflammatory cell infiltrations. PRP group displayed evidenced healing process via proliferation of the covering stratified epithelium to close the ulcer margins and covered the underlying connective tissue stroma which formed from large number of fibroblasts, collagen bundles, and newly formed blood vessels (Figure 1 D, E, F). 
At $10^{\text {th }}$ day in control group, the healing process appeared by prominent proliferation of the covering epithelium to close the ulcer margin as well as the connective tissue stroma that showed fibroblasts, collagen bundles, newly formed blood vessels and few inflammatory cells. HA group revealed complete reorganization of the normal epithelial stratification with normal architecture of the underlying lamina propria, as well as PRP group showed complete epithelialization of the ulcerative area with normal stratification, reorganization of the epithelium and the underlying connective tissue (Figure $1 \mathrm{G}, \mathrm{H}, \mathrm{I}$ ).

\section{CD 31 immunohistochemical analysis}

The area percentage of a positive reaction for CD31 in the experimental groups at $3^{\text {rd }}$ day post ulcer induction revealed significant increase of mean \pm SD results at HA group (0.56 \pm .019$)$, PRP group $(0.72 \pm .028)$ in comparison to control group $(0.25 \pm .032)$. Also $7^{\text {th }}$ day showed significant increase

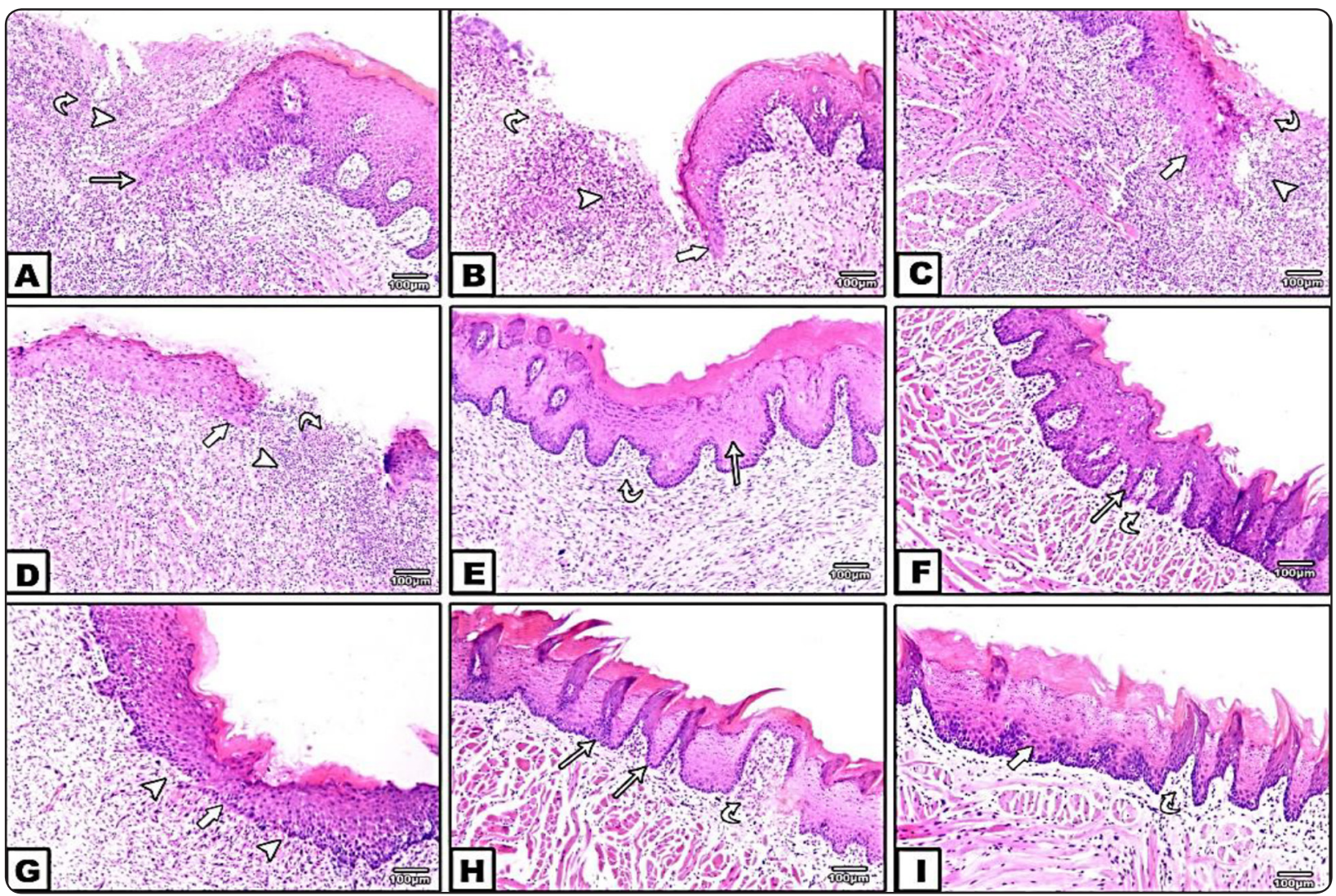

Fig. (1): photomicrograph showed the ulcer area at 3rd day (A, B and C) control,HAand PRP groups, all experimental groups revealed discontinuity of the epithelium (arrow) with formation of the granulation tissue (arrow head) and sever inflammatory cell infiltration (curved arrow). At 5th days (D) control group showed slight proliferation of the covering epithelium (arrow) and formation of the granulation tissue (arrow head) with dense inflammatory cell infiltration (curved arrow); (E, F) HA,PRP groups showed the beginning of The healing process was evident as the covering stratified epithelium proliferated to close the ulcer margins (arrow) and the covered the underlying connective tissue formed from large number of fibroblasts, collagen bundles, and newly formed blood vessels (curved arrow). At 10th day (G) control group revealed prominent proliferation of the covering epithelium (arrow) to close the ulcer margin as well as the connective tissue formed of newly formed blood vessels and few inflammatory cells (arrow head); (H, I) HA, PRP groups revealed complete reorganization of the normal epithelial stratification (arrow) with normal architecture of the underlying lamina propria (curved arrow) (H\&E stain, $\mathrm{x}$ 100) 
of mean \pm SD results at HA group (1.8 \pm 0.03$)$, PRP group $(2.07 \pm 0.42)$ in comparison to control group $(1.13 \pm 0.15)$. After $10^{\text {th }}$ days of ulcer induction revealed showed significant difference of mean \pm $\mathrm{SD}$ results at HA group (1.35 \pm 0.026$)$, PRP group $(1.24 \pm 0.01)$ in comparison to control group $(1.10 \pm$ $0.01)$ figure (2).

Statistically, ANOVA test revealed an overall significant difference between all groups during all examination periods. Moreover, Posthoctukey test for multiple comparisons at $3^{\text {rd }}$ and $7^{\text {th }}$ day showed significant difference $(P<0.0001)$ between control group and HA, PRP groups conversely.
Also, there was a significant difference between HA $\&$ PRP groups $(P<0.0001)$. At $10^{\text {th }}$ day displayed significant difference $(\mathrm{P}<0.0001)$ between control group and HA \& PRP groups. Also, there was significant difference $(P<0.0001)$ between HA and PRP groups Table (1).

\section{Ki-67 immunohistochemical analysis}

The Ki-67 expression was detected in the oral epithelium and was restricted to the basal and parabasal layers of the epithelium with parabasal layer showing intense staining. Quantitative statistical analysis for the positive nucleus stain displayed at $3^{\text {rd }}$ day post ulcer induction revealed
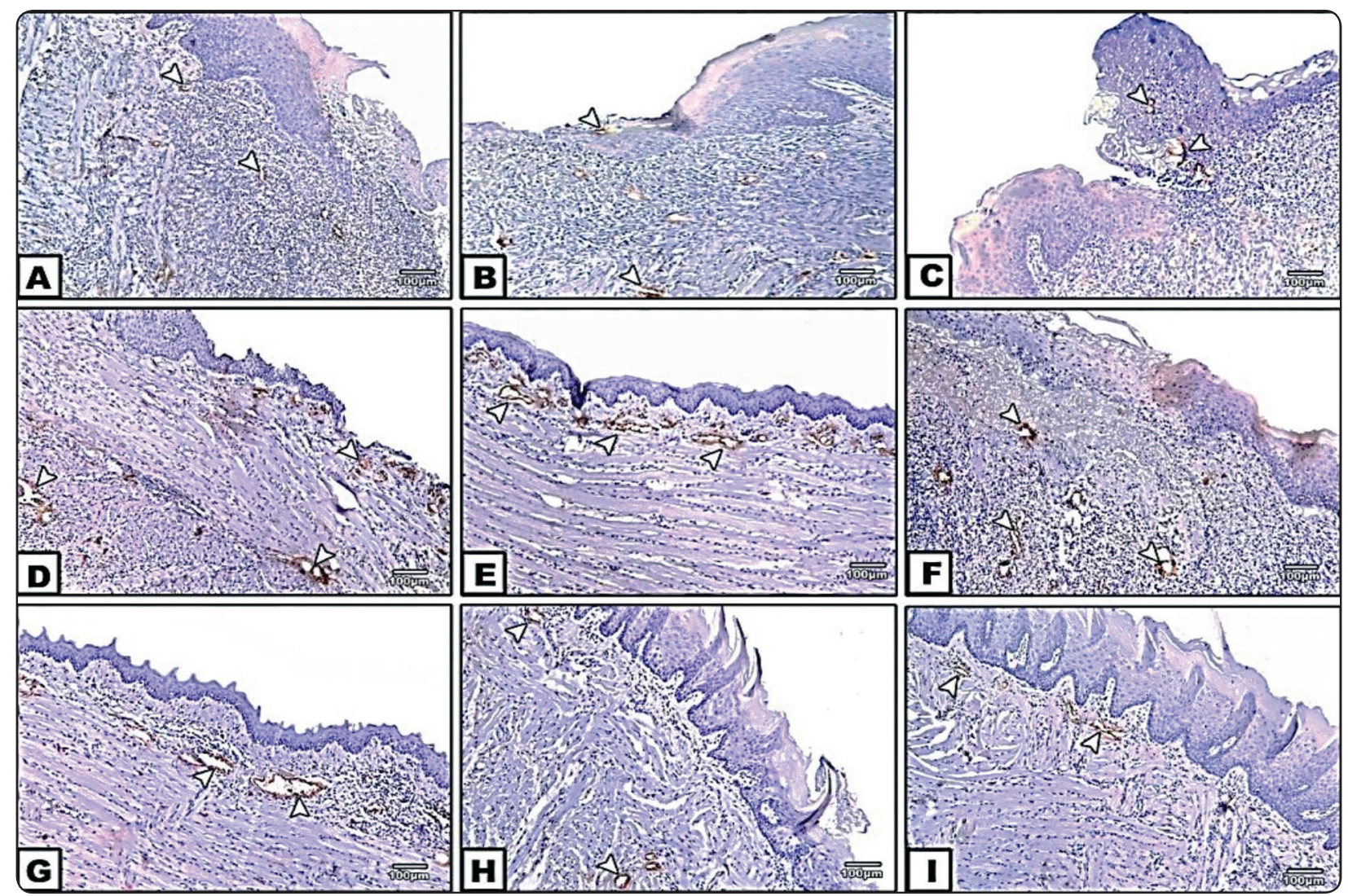

Fig. (2): Immunohistochemical expression of CD31 in the cytoplasm and cell membrane of endothelial cells and stromal connective tissue cells (arrow head). (A, B, and C): control, HA and PRP group at day 3. (D, E, and F): control, HA and PRP group at day 7. (G, H and I): control, HA and PRP group at day 10. (IHC staining, CD31 DAB chromagen X100). (IHC staining, CD31 DAB chromagen X100). 
significant increase of mean \pm SD results at HA group $(422.88 \pm 9.34)$, PRP group $(438.13 \pm 8.95)$ in comparison to control group (347.88 \pm 3.56$)$. Also $7^{\text {th }}$ day showed significant decrease of mean \pm SD results at HA group (186.13 \pm 5.84$)$, PRP group $(196.00 \pm 2.77)$ in comparison to control group $(165.00 \pm 3.20)$. After $10^{\text {th }}$ days of ulcer induction showed significant decrease of mean \pm SD results at HA group (95.00 \pm 4.00$)$, PRP group (4.46 \pm 89.75$)$ in comparison to control group $(110.13 \pm 5.43)$, figure (3).
ANOVA test revealed an overall significant difference between all groups during all examination periods. Moreover, Posthoctukey test for multiple comparisons along examination periods showed significant difference $(P<0.0001)$ between control group and HA \& PRP group. Also, there was a significant difference $(P<0.0001)$ between HA \& PRP groups at $3^{\text {rd }}$ and $7^{\text {th }}$ day. However, there was non- significant difference between HA and PRP groups at $10^{\text {th }}$ day $(\mathrm{P}=0.996)$ Table (2).
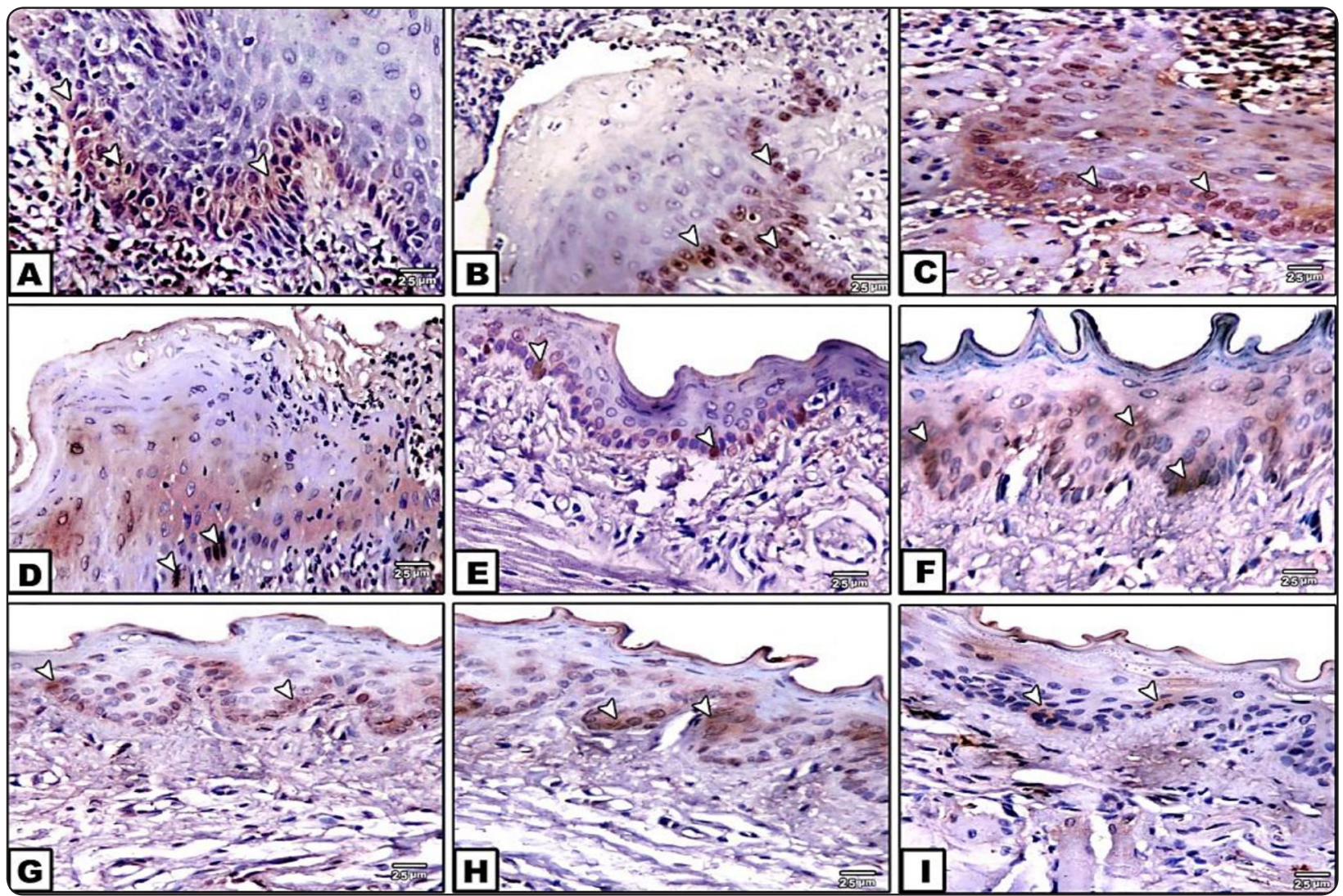

Fig. (3): Immunohistochemical expression of Ki67 as positive stain in the nucleus of basal and parabasal layers of the epithelium (arrow head). (A, B, and C): control, HA and PRP group at day 3. (D, E, and F): control, HA and PRP group at day 7. (G, $\mathrm{H}$ and I): control, HA and PRP group at day 10. (IHC staining, Ki 67 DAB chromagen X400). (IHC staining, Ki 67 DAB chromagen X400). 
TABLE (1): Shows means \pm STD of the CD31 expression of the studied groups at three-examination period.

\begin{tabular}{|c|c|c|c|c|}
\hline \multirow{2}{*}{ Periods } & \multicolumn{3}{|c|}{ Mean \pm STD of CD31 } & \multirow{2}{*}{$\mathbf{P}$} \\
\hline & Control Group & HA Group & PRP Group & \\
\hline $3^{\text {rd }}$ day & $0.25 \pm .032$ & $0.56 \pm .019$ & $0.6 \pm .023$ & \multirow{3}{*}{$<0.0001$} \\
\hline P1 & & $<0.0001$ & $<0.0001$ & \\
\hline $\mathbf{P 2}$ & & & 0.01 & \\
\hline $5^{\text {th }}$ day & $1.13 \pm 0.15$ & $1.8 \pm 0.03$ & $1.95 \pm 0.03$ & \multirow{3}{*}{$<0.0001$} \\
\hline P1 & & $<0.0001$ & $<0.0001$ & \\
\hline $\mathbf{P 2}$ & & & 0.01 & \\
\hline $10^{\text {th }}$ days & $1.10 \pm 0.01$ & $0.01 \pm 1.24$ & $0170 . \pm 1.22$ & \multirow{3}{*}{$<0.0001$} \\
\hline P1 & & $<0.0001$ & $<0.0001$ & \\
\hline $\mathbf{P 2}$ & & & 0.059 & \\
\hline
\end{tabular}

Data expressed by using mean \pm SD for 5 replica each

STD: standard deviation P: Probability

Test used: ANOVA followed by post Hoc test for multiple comparisons

P1: Significance relative to Group I

P2: Significance relative to Group II

TABLE (2): Shows means \pm STD of the KI 67 expression of the studied groups at three-examination period.

\begin{tabular}{|c|c|c|c|c|}
\hline \multirow{2}{*}{ Periods } & \multicolumn{3}{|c|}{ Mean \pm STD of KI 67} & \multirow{2}{*}{$\mathbf{P}$} \\
\hline & Control Group & HA Group & PRP Group & \\
\hline $3^{\text {rd day }}$ & $347.88 \pm 3.56$ & $422.88 \pm 9.34$ & $428.13 \pm 10.56$ & \multirow{3}{*}{$<0.0001$} \\
\hline $\mathbf{P 1}$ & & $<0.0001$ & $<0.0001$ & \\
\hline $\mathbf{P 2}$ & & & 0.438 & \\
\hline $5^{\text {th }}$ day & $7.63 \pm 176.75$ & $191.13 \pm 5.96$ & $196.00 \pm 2.77$ & \multirow{3}{*}{$<0.0001$} \\
\hline P1 & & $<0.0001$ & $<0.0001$ & \\
\hline $\mathbf{P 2}$ & & & 0.237 & \\
\hline $10^{\text {th }}$ days & $110.13 \pm 5.43$ & $4.00 \pm 95.00$ & $4.46 \pm 89.75$ & \multirow{3}{*}{$<0.0001$} \\
\hline $\mathbf{P 1}$ & & $<0.0001$ & $<0.0001$ & \\
\hline $\mathbf{P 2}$ & & & 0.86 & \\
\hline
\end{tabular}

Data expressed by using mean \pm SD. for 5 replicas each

STD: standard deviation P: Probability

Test used: ANOVA followed by post Hoc test for multiple comparisons

P1: Significance relative to Group I

P2: Significance relative to Group II 


\section{DISCUSSION}

Ulcer healing is a scheduled biologic process of cell migration and proliferation, alongside the extracellular matrix deposition, angiogenesis, and remodeling. Concerning the animal model for our work, the rodent tongue was the model of choice, as it affords the greatest reproducibility and sufficient tissue sampling. Also, the dorsal surface of the tongue covered with keratinized epithelium and nonkeratinized epithelium and can express the epithelial tissues of oral mucosa ${ }^{(24)}$.

The oral ulcers in present study were induced by using biopsy punch to form deep tissue defect and additional consuming the formocresol was providing chronic microenvironment to the ulcer. At present there are numerous topical agents for the management of oral ulcers such as steroids, antibiotics, analgesics and natural herbal medicines, the effectiveness of topical hyaluronic acid has been verified in substantial decline the symptoms ${ }^{(25)}$. Platelet-rich plasma (PRP), a platelet concentrate contained in a minor amount of plasma, has develop an optimistic treatment for many diseases associated with the skin owing to its great application of growth factors. It is an ideal and not dangerous therapy for the patient because of its autologous source, it reduces the possibility of adverse immune responses and transfusion-transmitted infections ${ }^{(26)}$.

High molecular weight HA is considered the most important proteoglycan found naturally in mucosal extracellular matrix and plays a predominant role in tissue morphogenesis, cell migration, differentiation, and adhesion. Above 1,000 kDa, HA is defined as high molecular weight HA (HMW-HA). HMWHA possesses anti-inflammatory, anti-proliferative, and anti-angiogenic properties and is, furthermore, involved in wound healing processes ${ }^{(27)}$.

In the present study, the histological findings of control group stained with $\mathrm{H} \& \mathrm{E}$ showed granulation tissue filling the ulcer area with inflammatory cell infiltrations at 3 days post ulceration. Newly formed epithelium appeared at 7 days till formation of complete epithelial layer covered the ulcerated area after 10 days. However, HA and PRP treated group revealed marked approximation of the ulcer edges and appeared mitotic activity with further migration of the epithelium into ulcer bed. The epithelial layers become more organized with well-structured and thickened epithelial strata throughout the second and third experimental periods.

The results approved that both PRP and HA can accelerate the healings of mucosal ulcers in comparison to control in all experimental periods except at the start of the experiment. In the initial stages following tissue damage, dominate neutrophils are carrying out phagocytosis, the neutrophils are gradually replaced by monocytes. Moreover, chemotaxis factors for neutrophils and monocytes are triggered throughout all phases of inflammation, keeping the immigration of monocytes subsequently to the movement of neutrophils. Roughly 3 days later, the histological outline of the ulcer begins to current modifications, the cellular elements convert mostly to lymphoplasmacytic infiltration, which is distinctive of chronic inflammation. The production of new blood vessels and many of fibroblasts occurs in this phase that indicating the start of repair ${ }^{(2)}$.

The superior healing advantages occurred in the PRP group compared to the HA one is related to the effect of the PRP on endogenous HA itself, as PRP helps in preserving the standard levels of endogenous $\mathrm{HA}$, and increases the HA biological properties. It is actually believed that both PRP and HA seems to match each other. The enhanced of ulcer healing and approximation of ulcer edges of PRP treated group was correspondingly reinforced challenges to convey growth factors to tissue defects consuming platelets have been established and exposed useful efficiency on chronic wound healing ${ }^{(28)}$.

Autologous PRP gel made from a patient's blood and comprises of cytokines, growth factors, chemokines, and a fibrin scaffold. PRP gel is believed to stimulate the normal wound healing responses by molecular and cellular induction like that happen 
with platelet triggering ${ }^{(29)}$. Amable et al., (2013) designed a study measuring the amount of growth factors, cytokines and chemokines secreted by the platelet concentrate after calcium activation. The reported high concentrations of platelet derived growth factor (PDGF), epidermal growth factor (EGF) and transforming growth factor (TGF) secreted together with pro and anti-inflammatory cytokines like interleukin 4 (IL-4), IL-8, IL-13, IL-17, tumor necrosis factor alpha (TNF $\alpha)$ and interferon alpha (INF $\alpha$ ). Also, there weren't any cytokine was secreted before platelet activation ${ }^{(30)}$. The stimulation induced by Platelet-released growth factors (PRGF) but not the PP-preparation supports the participation of platelet-secreted factors on HA synthesis. In fact, a stimulatory action of TGF-1 and PDGF, which are major platelet secretory products had been previously reported. Particularly the former up-regulates the hyaluronan synthase isoform 1 while PDGF primarily stimulates hyaluronan synthase isoform ${ }^{(31)}$.

Topical HA gel forms a protective coating around the oral cavity to shield exposed or sensitized nerve endings from overstimulation. Also, it enhances tissue hydration, and accelerates healing. Patients treated with topical HA experienced an immediate and sustained reduction of symptoms such as pain from ulcerated area and an improvement in oral function particular in the eating ${ }^{(32)}$.

Hyaluronic acids are polysaccharides that released normally through connective, epithelial, and neural tissues. The favorable influence of hyaluronic acid in wounds healing may be attributed to the enhanced water retention, which favors an appropriate environment for the formation of collagen and elastin, and permits the cells to proliferate and differentiate, accelerating the healing process. HA also affords two significant roles during normal healing processes. First, HA can assist the flow of nutritional materials and aids the wound to get rid of unwanted yields from cell metabolism. Second, HA is faithfully promoting keratinocyte proliferation and migration. The major receptor for HA on the surface of epidermal keratinocytes is cluster determinant 44 (CD44). Activation of CD44 by HA increases signal transduction and several downstream biological activities, additionally, the anti-inflammatory properties of hyaluronic acid control the healing, inhibiting the conversion of wound ${ }^{(33)}$.

Treatment with PRP potentially could have an anti-inflammatory effect on soft tissues through the release of vital growth factors in both acute and chronic cases. In addition, there are no negative effects of reactive oxygen species, proteolytic enzymes, or matrix metalloproteinases released by WBCs when PRP is used. However HAcan stimulate wound healing through HA-CD44 interaction that induces intracellular signal cascades, regulates cell-cell adhesion, cell migration, and proliferation required wound healing ${ }^{(34)}$.

Angiogenesis during healing processes shows a significant role in developing the granulation tissue and refining the wound vasculature. CD44 plays a significant role in the maintenance of central nervous system (CNS) microvascular barrier integrity. The role of CD44 in mediating its modulation of vascular barrier integrity via regulation of PECAM-1 (plateletendothelial cell adhesion molecule) and (CD31) expression, specifically by CD31 modulation of endothelial cell junctional integrity. Tsuneki M \& Madri JA (2014) approved the roles of CD44 in modulating both proliferation and apoptosis of microvascular endothelial cells via its modulation of CD31 and VE-cadherin expression via Hippo pathway activation ${ }^{(34)}$.

CD31 immune-reactivity was identified in the endothelial cells of the blood vessels and some of stromal cells. The statistical results of the current work showed significant increase in CD31 immunoexpression in the granulation tissues of PRP group in comparison to control group. These outcomes were in agreement with those of Zhang et al., (2018) that PRP treatment significantly improved the hemorheological indexes, decreasing the blood viscosity, plasma viscosity, hematocrit and erythro- 
cyte aggregation in the rabbit model of avascular necrosis of the femoral head. PRP can promote angiogenic growth factors affects angiogenesis. Also, micro particles originated from platelet can induce proliferation, survival, adhesion, and chemotaxis of hematopoietic cells in vitro. The current results are in constituent with earlier studies and reveal that PRP stimulates the proliferation of endothelial cells and correspondingly can promote the formation of endothelial cells ${ }^{(35)}$.

Statistical analysis of CD31 as one of angiogenic markers displayed significant increase in HA treated group compared to control group. HA shows a pivotal role in the development of embryonic/ neonatal tissue, offers organizational and cellsignaling signals, keeps tissue homeostasis, and controls the formation and progress of several uncontrolled disorders due to the abnormalities of angiogenesis. Authors described that hyaluronan (HA) degradation products can enhance formation of blood vessels in vivo, and affect endothelial cell invasion of a 3-dimensional matrix, which is a necessary constituent during neovascularization. In addition, they suggested that the synergism between (HA) degradation products and VEGF acts as a guideline for angiogenesis and stimulates the growth of new blood vessel ${ }^{(36)}$.

The expression of CD31 was decreased at $10^{\text {th }}$ day post ulcer induction. These findings were in agreement with Ciccone et al., (2019) that the neovascular response was obvious in the first week before undergoing regression. VEGF and FGF-2 levels were high for the first week, while the overall balance of the angiogenesis modulators moved toward inhibition after one week with decreased VEGF and FGF-2 levels and increased expression of endostatin ${ }^{(37)}$. Moreover, other authors suggested that a release of VEGF from epidermal cells and macrophages prompts and maintains early angiogenic steps by the $2^{\text {nd }}$ and $3^{\text {rd }}$ days after wounding. Highest action arises through nearly 3 to 7 days later to wound. As soon as the granulation tissue is formed, angiogenesis ceases and blood vessels decline ${ }^{(38)}$.

Regarding statistical analysis of cellular proliferation using Ki67 revealed a significant increase in HA, PRP groups in comparison to control group among the examination periods, that comes in agreement with Vaquerizo et al.,(2018) that superior clinical perfection in treatment of knee osteoarthritis, occurred in the PRP group compared to the HA one, which is attributed to the influence of the PRP on endogenous HA itself, as PRP helps in restoring the normal levels of endogenous HA which is considered as the central synovial fluid constituent and is intensely reduced in the degenerative joint diseases, also PRP increases the HA biological properties ${ }^{(8)}$. We actually believe that both PRP and HA appears to complement each other.

Growth factors support fast proliferation of numerous undifferentiated mesenchymal cells at wound area during the regenerative process. So, the PRP benefit is to promote the healing mechanism by increasing the amount of growth factors existing in the platelets. Various studies have shown that both platelet-rich plasma (PRP) and platelet-rich fibrin (PRF) promote settlement, adhesion, proliferation and migration of cells due to high concentrations of growth factors like PDGF, TGF and IGF leading to improved wound healing ${ }^{(39)}$.

HA gel was recognized in regulating tissue hydration during the times of inflammation process. This increases cell proliferation and migration. It leads to decline of cell attachment to the extra cellular matrix and permit short-term detachment to help the cellular migration and division. In the hydrated state, copious of water surround the HA molecule is obstructed which leads to restriction of water movement and minor molecules. Moreover, HA is viscous which decreases secondary infection by viral and bacterial attack. In addition, HA may have a reasonable influence over free-radical scavenging, antioxidant effect, and exclusion of tissue degrading enzyme during inflammation ${ }^{(37)}$. Many functions of hyaluronan depend upon its interaction with 
cell surface receptors, including CD44. CD44mediated cell interaction with hyaluronan has been implicated in a variety of physiological events including cell-cell and cell-substrate adhesion, cell migration, cell proliferation, and hyaluronan uptake and degradation ${ }^{(40)}$.

\section{CONCLUSION}

The findings of the obtained results indicated that the benefits of topical application of PRP is not only an effective and safe in the treatment of oral ulcers, but also is a significant better than HA treatment. PRP may therefore be a viable alternative or an adjuvant therapy to standard existing drugs used for treatment of oral ulcers. However, before suggesting this procedure to patients, more clinical trials are needed to confirm the conclusions.

\section{REFERENCES}

1. Guo S, DiPietro LA. Critical review in oral biology \& medicine: Factors affecting wound healing. Journal of Dental Research. 2010;89(3):219-229.

2. I GMC, Janaína R, Paula S De, Peres L, Ii DS, Bitu F, et al. Alimentary Tract Experimental model of traumatic ulcer in the cheek mucosa of rats 1 Modelo experimental de úlcera traumática em mucosa jugal de ratos. Acta Cirúrgica Brasileira -. 2011;26(3):227-234.

3. Ialenti A, Di Rosa M. Hyaluronic acid modulates acute and chronic inflammation. Agents and Actions. 1994;43(12):44-47.

4. Bohaumilitzky L, Huber AK, Stork EM, Wengert S, Woelfl F, Boehm H. A trickster in disguise: Hyaluronan's ambivalent roles in the matrix, Frontiers in Oncology. 2017;7:242.

5. Chen M, Li L, Wang Z, Li P, Feng F, Zheng X. High molecular weight hyaluronic acid regulates $P$. gingivalisinduced inflammation and migration in human gingival fibroblasts via MAPK and NF- $\varkappa$ B signaling pathway. Archives of Oral Biology. 2019;98:75-80.

6. Badiu D, Vasile M, Teren O. Regulation of wound healing by growth factors and cytokines. Wound Healing: Process, Phases and Promoting. 2011;73-93.

7. Nikolidakis D, Jansen JA. The biology of platelet-rich plasma and its application in oral surgery: Literature review. Tissue Engineering - Part B: Reviews. 2008;14(3):249-258.
8. Vaquerizo V, Padilla S, Aguirre JJ, Begoña L, Orive G, Anitua E. Two cycles of plasma rich in growth factors (PRGF-Endoret) intra-articular injections improve stiffness and activities of daily living but not pain compared to one cycle on patients with symptomatic knee osteoarthritis. Knee Surgery, Sports Traumatology, Arthroscopy. 2018;26(9):2615-2621.

9. Cheng B, Fu X. The Focus and Target: Angiogenesis in Refractory Wound Healing. International Journal of Lower Extremity Wounds. 2018;17(4):301-303.

10. Pusztaszeri MP, Seelentag W, Bosman FT. Immunohistochemical expression of endothelial markers CD31, CD34, von Willebrand factor, and Fli-1 in normal human tissues. Journal of Histochemistry and Cytochemistry. 2006;54(4):385-395.

11. Vieira FLD, Vieira BJ, Guimaraes MAM, Aarestrup FM. Cellular profile of the peritumoral inflammatory infiltrate in squamous cells carcinoma of oral mucosa: Correlation with the expression of Ki67 and histologic grading. BMC Oral Health. 2008;8(1):1-8.

12. Luukkaa H, Klemi P, Leivo I, Vahlberg T, GréNman R. Prognostic significance of Ki-67 and p53 as tumor markers in salivary gland malignancies in Finland: An evaluation of 212 cases. Acta Oncologica. 2006;45(6):669-675.

13. Rashed FM, GabAllah OM, AbuAli SY, Shredah MT. The effect of using bone marrow mesenchymal stem cells versus platelet rich plasma on the healing of induced oral ulcer in albino rats. International Journal of Stem Cells. 2019;12(1):95-106

14. Alio JL, Abad M, Artola A, Rodriguez-Prats JL, Pastor S, Ruiz-Colecha J. Use of autologous platelet-rich plasma in the treatment of dormant corneal ulcers. Ophthalmology. 2007;114(7):1286-1293.

15. EL-Komy MH, Hassan AS, Raheem HM, Doss SS, ELKaliouby M, Saleh NA, Saleh MA. Platelet-rich plasma for resistant oral erosions of pemphigus vulgaris: A pilot study. Wound Repair and Regeneration. 2015 Nov 12;23(6):953-5.

16. Cieslik-Bielecka A, Skowroński R, Jędrusik-Pawłowska $\mathrm{M}$, Pierchała M. The application of L-PRP in AIDS patients with crural chronic ulcers: A pilot study. Advances in medical sciences. 2018 Mar 1;63(1):140-146.

17. Ahmed EM, Ali S, Gaafar SM, Rashed LM, Fayed HL. Evaluation of topical human platelet lysate versus topical clobetasol in management of methotrexate-induced oral 
ulceration in rheumatoid arthritis patients: Randomizedcontrolled clinical trial. International immunopharmacology. 2019;73 (8):389-394.

18. Ramzy MM, Essawy TA, Shamaa A, Mohamed SS. Evaluation of the Effect of Platelet Rich Plasma on Wound Healing in the Tongue of Normal and Streptozotocin-induced Diabetic Albino Rats: Histological, Immunohistochemical, and Ultrastructural Study. Open Access Macedonian Journal of Medical Sciences. 2020;8(9):666-689.

19. Del Fante C, Perotti C, Bonferoni MC, Rossi S, Sandri G, Ferrari F, et al. Platelet lysate mucohadesive formulation to treat oral mucositis in graft versus host disease patients: A new therapeutic approach. AAPS PharmSciTech. 2011;12(3):893-899.

20. Bartoš V, Adamicová K, Kullová M, Péč M. Immunohistochemical evaluation of proliferative activity (Ki-67 index) in different histological types of cutaneous basal cell carcinoma. Biologia. 2012;67(3):610-615.

21. Inomata T, Miwa Y, Kawata S, Omotehara T, Sato I, Itoh M. Immunohistochemical study for relationship between vessel and lymphatic properties and tooth marks in human oral mucosa. European Journal of Histochemistry. 2020;64(1).

22. Teófilo CR, Ferreira AEC, Batista AC, Jamacaru FVF, Sousa FB, Mota MRL, et al. Mast cells and blood vessels profile in oral carcinogenesis: An immunohistochemistry study. Asian Pacific Journal of Cancer Prevention. 2020;21(4):1097-10102.

23. Eming SA, Martin P, Tomic-Canic M. Wound repair and regeneration: Mechanisms, signaling, and translation. Science Translational Medicine. 2014;6(265):1-36.

24. Elsaadany B, El Kholy S, El Rouby D, Rashed L, Shouman T. Effect of Transplantation of Bone Marrow Derived Mesenchymal Stem Cells and Platelets Rich Plasma on Experimental Model of Radiation Induced Oral Mucosal Injury in Albino Rats. International Journal of Dentistry. 2017;2017.

25. Gupta S, Kediege SD, Gupta A, Jain K. Evaluation of gengigel ${ }^{\circledR}$ application in the management of furcation with coronally advanced flap through surgical re-entry-a split mouth clinical study. Journal of Clinical and Diagnostic Research. 2017;11(1):ZC27-32.

26. Monslow J, Govindaraju P, Puré E. Hyaluronan - a functional and structural sweet spot in the tissue microenvironment. Frontiers in Immunology. 2015;6(5):4-10.
27. Viola M, Vigetti D, Karousou E, D’Angelo ML, Caon I, Moretto P, et al. Biology and biotechnology of hyaluronan. Glycoconjugate Journal. 2015;32(3-4):93-103.

28. Casale M, Moffa A, Vella P, Sabatino L, Capuano F, Salvinelli B, et al. Hyaluronic acid: Perspectives in dentistry. A systematic review. International Journal of Immunopathology and Pharmacology. 2016;29(4):572-82.

29. Merchán WH, Gómez LA, Chasoy ME, Alfonso-Rodríguez CA, Muñoz AL. Platelet-rich plasma, a powerful tool in dermatology. Journal of Tissue Engineering and Regenerative Medicine. 2019;13(5):892-901

30. Amable PR, Carias RBV, Teixeira MVT, Da Cruz Pacheco Í, Corrêa Do Amaral RJF, Granjeiro JM, et al. Platelet-rich plasma preparation for regenerative medicine: Optimization and quantification of cytokines and growth factors. Stem Cell Research and Therapy. 2013;4(3):1-13.

31. Anitua E, Sánchez M, Nurden AT, Zalduendo MM, De la Fuente M, Azofra J, et al. Platelet-released growth factors enhance the secretion of hyaluronic acid and induce hepatocyte growth factor production by synovial fibroblasts from arthritic patients. Rheumatology. 2007;46(12):176972.

32. Dalessandri D, Zotti F, Laffranchi L, Migliorati M, Isola G, Bonetti S, et al. Treatment of recurrent aphthous stomatitis (RAS; Aphthae; canker sores) with a barrier forming mouth rinse or topical gel formulation containing hyaluronic acid: A retrospective clinical study. BMC Oral Health. 2019;19(1):1-10.

33. Bourguignon LYW, Ramez M, Gilad E, Singleton PA, Man MQ, Crumrine DA, et al. Hyaluronan-CD44 interaction stimulates keratinocyte differentiation, lamellar body formation/ secretion, and permeability barrier homeostasis. Journal of Investigative Dermatology. 2006;126(6):1356-65.

34. Tsuneki M, Madri JA. CD44 regulation of endothelial cell proliferation and apoptosis via modulation of CD31 and VE-cadherin expression. Journal of Biological Chemistry. 2014 Feb 28;289(9):5357-70.

35. Zhang XL, Shi KQ, Jia PT, Jiang LH, Liu YH, Chen X, et al. Effects of platelet-rich plasma on angiogenesis and osteogenesis-associated factors in rabbits with avascular necrosis of the femoral head. European Review for Medical and Pharmacological Sciences. 2018;22(7):2143-52.

36. Pardue EL, Ibrahim S, Ramamurthi A. Role of hyaluronan in angiogenesis and its utility to angiogenic tissue engineering. Organogenesis. 2008;4(4):203-14. 
37. Ciccone V, Zazzetta M, Morbidelli L. Comparison of the Effect of Two Hyaluronic Acid Preparations on Fibroblast and Endothelial Cell Functions Related to Angiogenesis. Cells. 2019;8(12).

38. Isner JM, Pieczek A, Schainfeld R, Blair R, Haley L, Asahara $\mathrm{T}$, et al. Clinical evidence of angiogenesis after arterial gene transfer of phVEGF165 in patient with ischaemic limb. Lancet. 1996;348(9024):370-374.
39. Steller D, Herbst N, Pries R, Juhl D, Hakim SG. Positive impact of Platelet-rich plasma and Platelet-rich fibrin on viability, migration and proliferation of osteoblasts and fibroblasts treated with zoledronic acid. Scientific Reports. 2019;9(1):1-11.

40. Solis MA, Chen YH, Wong TY, Bittencourt VZ, Lin YC, Huang LLH. Hyaluronan regulates cell behavior: A potential niche matrix for stem cells. Biochemistry Research International. 2012;2012. 